\title{
A case report: Bilateral atraumatic proximal tibiofibular joint osteoarthritis
}

\author{
Gilberto Yoshinobu Nakama ${ }^{1,2^{*}}$, Guilherme C. Gracitelli ${ }^{1,2}$, Alberto de Castro Pochini $^{2}$, \\ Caio Augusto de Souza Nery ${ }^{2}$, Mario Carneiro Filho ${ }^{1,2}$ \\ ${ }^{1}$ Knee Surgery and Arthroscopy Group, Federal University of São Paulo, São Paulo, Brazil; \\ *Corresponding Author: gilberto nakama@yahoo.com.br \\ ${ }^{2}$ Department of Orthopedics and Traumatology, Federal University of São Paulo, São Paulo, Brazil
}

Received 20 April 2013; revised 21 May 2013; accepted 15 June 2013

Copyright (C) 2013 Gilberto Yoshinobu Nakama et al. This is an open access article distributed under the Creative Commons Attribution License, which permits unrestricted use, distribution, and reproduction in any medium, provided the original work is properly cited.

\begin{abstract}
Introduction: Proximal tibiofibular joint osteoarthritis is a rare disease most commonly occurring in the presence of either systemic inflammatory conditions or severe knee osteoarthritis. Case Presentation: The authors present a case report of isolated bilateral tibiofibular arthrosis in an otherwise healthy 28-year-old female patient. The patient presented with complaints of lateral knee pain. Radiographs and magnetic resonance imaging (MRI) revealed bilateral tibiofibular joint osteoarthritis. Trauma, repetitive use injury, and rheumatologic causes were excluded. The patient's pain was initially managed conservatively, but she subsequently underwent tibiofibular arthrodesis for progressive pain symptoms. We review in this report the current literature on tibiofibular joint osteoarthritis and its treatment. Conclusion: Isolated tibiofibular joint osteoarthritis is a rare condition that may progress and requires surgical treatment to achieve optimal clinical outcomes.
\end{abstract}

Keywords: Proximal Tibiofibular Joint; Osteoarthritis; Arthrodesis

\section{INTRODUCTION}

Proximal tibiofibular joint pathology is increasingly being recognized as a cause of lateral knee pain, particularly in young adults and athletes $[1,2]$. Due to the mild, nonspecific nature of early symptoms, the majority of diagnoses are made in the later phases of disease progression, particularly where instability exists in the proximal tibiofibular joint [3]. Rare case reports describe bilateral idiopathic tibiofibular joint arthritis [4] and atraumatic tibiofibular joint instability [5], but most cases of tibiofibular joint arthritis occur in the presence of predisposing conditions such as generalized joint laxity [6], systemic inflammatory disease [7], and severe knee osteoarthritis [8]. This article reports an unusual case of bilateral tibiofibular arthritis in an otherwise healthy 28year-old sedentary female.

\section{CASE REPORT}

A 28-year-old woman presented to our clinic with a history of two months of bilateral lateral-sided knee pain, gradually progressive and worse on the left side than the right. She reported no preceding injury and described her activity level as sedentary, never having participated in any regular sports or fitness activities. She was not obese, with a body mass index (BMI) of 24. She denied any history of lower extremity injury or pain prior to her current symptoms. She reported no history of any other medical problems. She reported that her pain symptoms were provoked by knee flexion and walking for long distances. Her Visual Analog Scale (VAS) pain score at presentation was four points. She reported substantial relief of her pain symptoms after taking common analgesics.

Clinical examination revealed a well-appearing patient in no acute distress. The face and extremities were without rashes or evidence of inflammation. Ligamentous laxity was within normal limits. Lower extremity limb lengths were equal, and the alignment of the lower extremities was physiologic. No erythema or effusion was noted for either knee joint. The proximal tibiofibular joints were tender to palpation bilaterally. Full, symmetric range of motion was present bilaterally for the hips, knees, and ankles without pain during examination. The 
passive joint play test for hypermobility [9] was negative bilaterally. Specific examinations for patellofemoral disorders and tendon, ligament, or meniscal injuries were negative.

Laboratory analyses revealed negative results for antinuclear factor, antinucleosome antibodies, rheumatoid factor, anti-cyclic citrullinated peptide antibody, and serum protein electrophoresis. Mild elevations were observed for c-reactive protein (14 mg/L) and erythrocyte sedimentation rate $(23 \mathrm{~mm} / \mathrm{Hr})$.

Lower extremity radiographs revealed bilateral proximal tibiofibular joint sclerosis and posterior osteophyte formation. In these imaging studies, the proximal tibiofibular joints were oblique, with greater than 20 degrees of inclination relative to horizontal plane bilaterally (Figures 1 and 2). Ankle radiographs revealed no associated distal tibiofibular joint pathology in either limbs. Magnetic resonance imaging of the knee joints demonstrated bilateral isolated proximal tibiofemoral joint cartilage degeneration and joint space narrowing (Figures 3 and 4).

The patient was initially managed conservatively with rest, ice, anti-inflammatory medication, and glucosamine supplements. She reported symptomatic improvement after 3 months of this treatment. Four years after her initial presentation, the patient presented again with in-

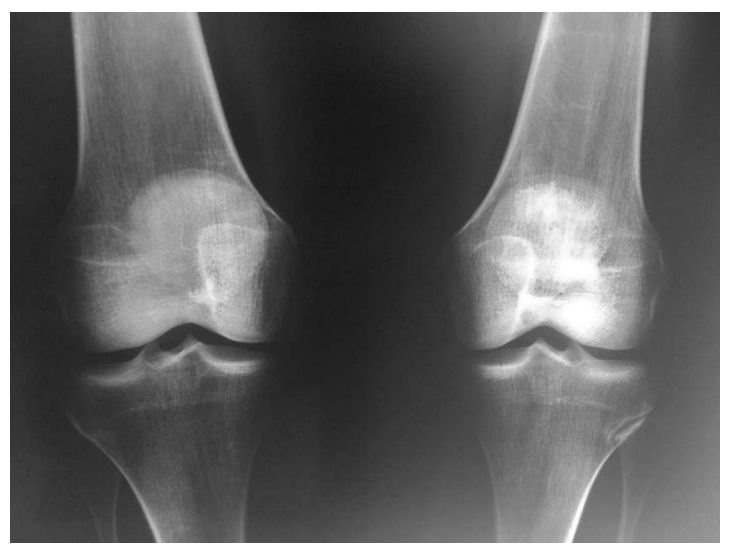

Figure 1. Radiographs AP view.

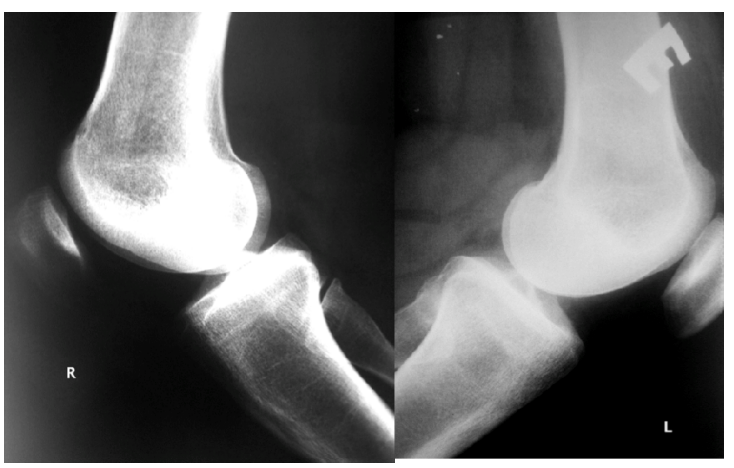

Figure 2. Radiographs lateral view.

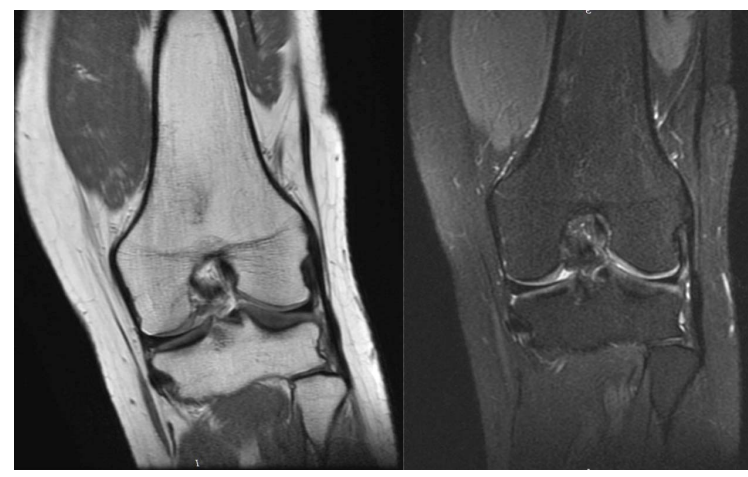

Figure 3. Magnetic resonance imaging of the knee joints.

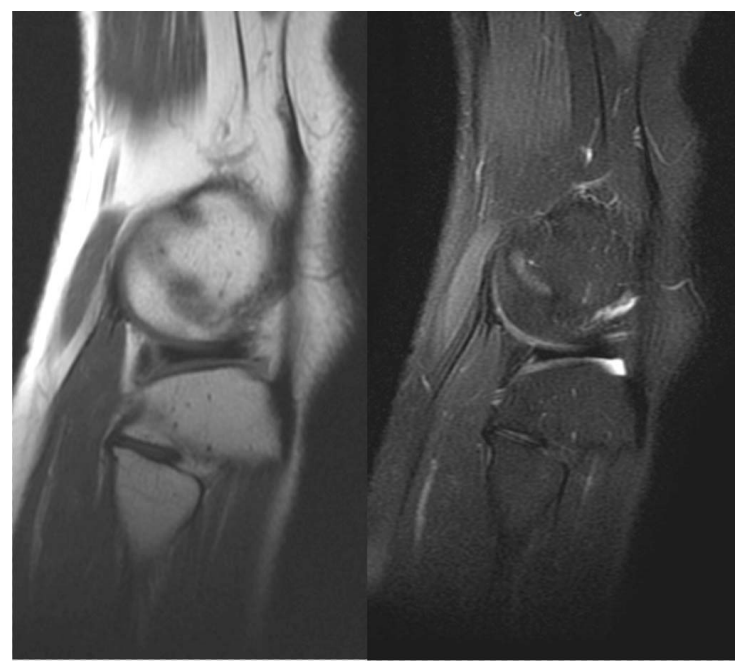

Figure 4. Magnetic resonance imaging of the knee joints.

creased lateral-sided knee pain which did not respond to conservative measures and which was again greater on the left side. At this time, she reported a VAS pain score of seven for her left knee.

Surgical treatment with left proximal tibiofibular arthrodesis was offered to the patient following the failure of conservative management, and the patient consented to undergo surgery. In this procedure, a curvilinear "hockey-stick" lateral incision was used for the approach (Figure 5). The peroneal nerve was isolated and protected. The tibiofibular joint was accessed (Figure 6). Following resection of the cartilage surfaces of the proximal tibiofibular joint, the surfaces were compressed using a 6.5-mm Arbeitsgemeinshaft fur Osteosynthesefragen (AO) partially threaded cancellous bone screw and washer. Radiographs confirmed satisfactory fixation (Figures 7 and 8).

Following surgery, the patient was immobilized in a long cast for 6 weeks without weight bearing. The patient was placed in a removable boot to be worn during ambulation and allowed to be weight-bearing as tolerated in this boot for an additional 4 weeks. The patient reported adequate pain control in her right knee with rest, 


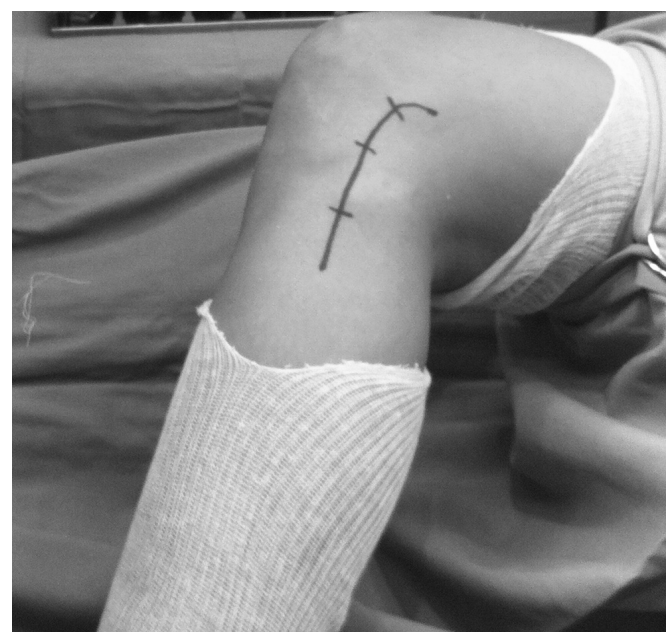

Figure 5. Curvilinear “hockey-stick” lateral incision.

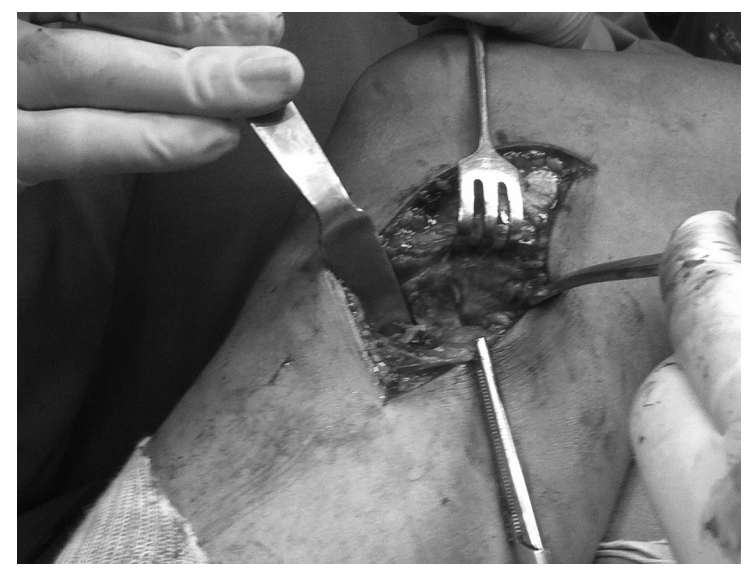

Figure 6. The tibiofibular joint acesss.

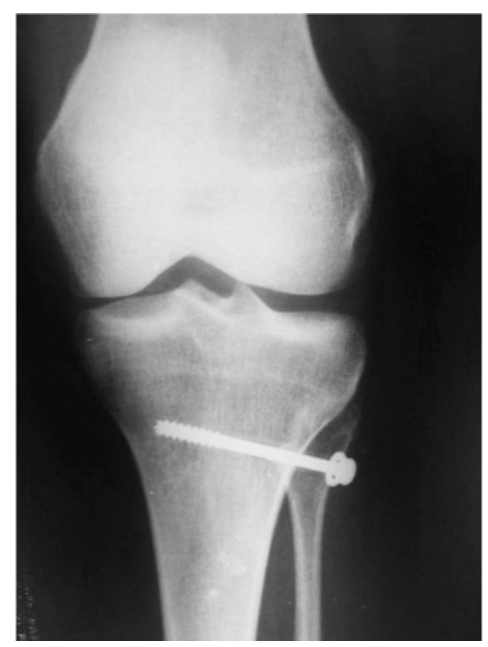

Figure 7. Radiographs after fixation.

ice, anti-inflammatory medication, and glucosamine and was therefore not offered surgical treatment for this extremity. Two years postoperatively, patient reports a VAS pain score of one and has full knee range of motion

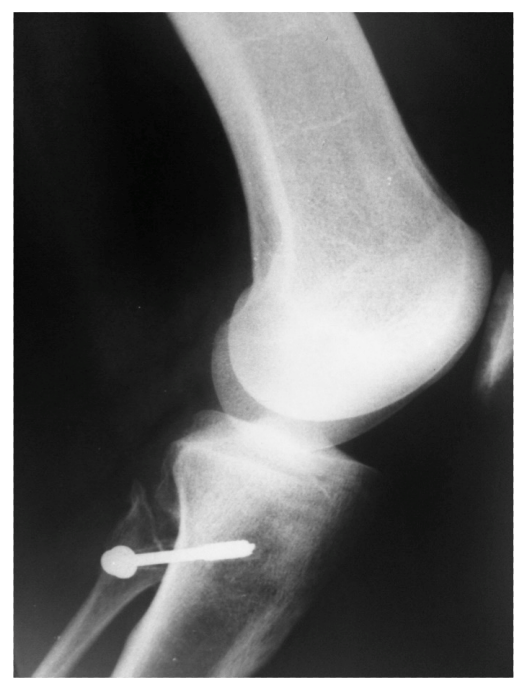

Figure 8. Radiographs after fixation.

with flexion to $140^{\circ}$ bilaterally.

\section{DISCUSSION}

Lateral knee pain is a common musculoskeletal complaint, and causes for this complaint in young patients may include lateral meniscal injury, patellofemoral disorders, iliotibial band syndrome, ligamentous injury, discoid meniscus, tumor, or infection. In older patients, degenerative arthritis becomes a more frequent cause of pain symptoms, as well, but patients presenting with degenerative changes are typically in their fifties, sixties, or older. The case presented here describes an occurrence of bilateral proximal tibiofibular joint degeneration in a young adult patient with no other underlying risk factors. In the literature, proximal tibiofibular joint arthritis has been reported most commonly in association with significant prior knee injury, systemic inflammatory disease, and severe tibiofemoral osteoarthritis [7,8]. In addition, degenerative changes in the proximal tibiofemoral joint may occur in young athletes following knee injuries $[10,11]$ or overload of the joint by repetitive sport-specific motions of the ankles or knees [12]. Idiopathic subluxation of the proximal tibiofibular joint may also be present and predispose to later joint pain and degeneration [10].

Ogden [1] has described two anatomic types of proximal tibiofibular joint inclinations. According to this classification, a Type 1 inclination is horizontal. A Type 2 inclination is characterized by an oblique inclination greater than $20^{\circ}$ relative to horizontal plane. Type 1 articulations possess relatively more rotatory mobility with greater surface area than Type 2 articulations. In his anatomic study of the proximal tibiofibular joint, Ogden also defined the joint's three basic functions: 1) dissipating stress applied to the ankle, 2) dissipating stress 
from lateral tibial bending moments, and 3) tensile weight-bearing. The decreased mobility of Type 2 articulations described by Ogden was confirmed by the results of Barnett et al. [13]. In this case series, 70\% of dislocations and subluxations of tibiofibular joint occurred in patients with Type 2 articulations, supporting a role for this joint in relieving torsional stress at the ankle.

In the case described here, the patient's radiographs revealed that she had Type 2 proximal tibiofibular joints. She denied any history of knee or ankle injury, pain, or instability prior to her presenting complaints. As a cause for her pathology, we propose that perhaps the Type 2 alignment sufficiently compromised the mechanics of the proximal tibiofibular joints so that degeneration of these joints occurred with stresses generated in her daily activities. Unfortunately, since the patient presented with advanced degenerative changes at her initial evaluation, it was not possible to assess the degree to which joint instability may have contributed to the development of her condition. Although the patient's pain symptoms initially responded to conservative treatment, her pain symptoms in the left lower extremity progressed, and she elected to undergo surgical treatment on this side.

Surgical options for the treatment of proximal tibiofibular arthritis are limited. Excision of the fibular head may result in knee instability as a result of removal of the insertions of the fibular collateral ligament and biceps femoris tendon [10]. For patients with instability without degenerative changes, ligamentous reconstruction may also be considered. Arthrodesis of the proximal tibiofibular joint is another surgical option for pain at this joint, with some authors advocating the excision of a segment of the fibular shaft at the time of arthrodesis to avoid limitation of ankle movement [14]. In this patient, we chose to perform only arthrodesis of the proximal tibiofibular joint without fibular osteotomy given the risk of complications for this additional procedure [14,15] and considering the activity demands of our sedentary patient. We would consider fibular osteotomy if ankle symptoms were reported, such as pain or decreased range of motion, but our patient has not been found to have any ankle complaints or limitations on follow up examination. In this case of bilateral proximal tibiofibular joint arthritis, proximal tibiofibular arthrodesis alone, following failure of conservative therapies on one side, has provided improvement in pain and functional symptoms for our patient.

\section{CONCLUSION}

Proximal tibiofibular joint pathology is a diagnosis to consider as a cause of lateral knee pain. Pain symptoms from proximal tibiofibular joint degeneration may re- quire surgical treatment for symptom relief if conservative therapies fail.

\section{REFERENCES}

[1] Ogden, J.A. (1974) The anatomy and function of the proximal tibiofibular joint. Clinical Orthopaedics and Related Research, 186-191.

[2] Semonian, R.H., Denlinger, P.M. and Duggan, R.J. (1995) Proximal tibiofibular subluxation relationship to lateral knee pain: A review of proximal tibiofibular joint pathologies. Journal of Orthopaedic \& Sports Physical Therapy, 21, 248-257.

[3] Turco, V.J. and Spinella, A.J. (1985) Anterolateral dislocation of the head of the fibula in sports. The American Journal of Sports Medicine, 13, 209-215. doi:10.1177/036354658501300401

[4] Nadaud, M.C. and Ewing, J.W. (2001) Proximal tibiofibular arthritis: An unusual cause of lateral knee pain. Orthopedics, 24, 397-398.

[5] Morrison, T.D., Shaer, J.A. and Little, J.E. (2011) Bilateral, atraumatic, proximal tibiofibular joint instability. Orthopedics, 34, 133.

[6] Owen, R. (1968) Recurrent dislocation of the superior tibio-fibular joint. A diagnostic pitfall in knee joint derangement. The Journal of Bone \& Joint Surgery, 50, 342-345.

[7] Hong, H.P., Chung, H.W., Choi, B.-K., Yoon, Y.C. and Choi, S.H. (2009) Involvement of the proximal tibiofibular joint in ankylosing spondylitis. Acta Radiologica, 50, 418-422. doi:10.1080/02841850902783338

[8] Oztuna, V., Yildiz, A., Ozer, C., Milcan, A., Kuyurtar, F. and Turgut, A. (2003) Involvement of the proximal tibiofibular joint in osteoarthritis of the knee. The Knee, 10, 347-349. doi:10.1016/S0968-0160(03)00004-8

[9] Sijbrandij, S. (1978) Instability of the proximal tibio-fibular joint. Acta Orthopaedica Scandinavica, 49, 621-626. doi:10.3109/17453677808993250

[10] Ogden, J.A. (1974) Subluxation and dislocation of the proximal tibiofibular joint. The Journal of Bone and Joint Surgery, 56, 145-154.

[11] Ogden, J.A. (1974) Subluxation of the proximal tibiofibular joint. Clinical Orthopaedics and Related Research, 192-197.

[12] Takai, S., Yoshino, N., Aso, S. and Hirasawa, Y. (2012) Symptomatic spur formation of bilateral proximal tibiofibular joints. Orthopedics, 24, 843-845.

http://eutils.ncbi.nlm.nih.gov/entrez/eutils/elink.fcgi?dbfr om=pubmed\&id $=11570453 \&$ retmode $=$ ref\&cmd=prlinks

[13] Barnett, C.H. and Napier, J.R. (1952) The axis of rotation at the ankle joint in men. Its influence upon the form of the talus and the mobility of the fibula. Journal of Anatomy, 86, 1-9.

[14] Wootton, J.R., Ashworth, M.J. and MacLaren, C.A. (1995) Neurological complications of high tibial osteotomyThe fibular osteotomy as a causative factor-A clinical and anatomical study. Annals of the Royal College of 
Surgeons of England, 77, 31-34.

[15] Miskovsky, S., Kaeding, C. and Weis, L. (2004) Proximal tibiofibular joint ganglion cysts: Excision, recurence, and joint arthrodesis. The American Journal of Sports Medicine, 32, 1022-1028. doi:10.1177/0363546503258706 